\title{
Evaluating the \\ New Information Technologies
}

\author{
Jere Johnston
}

University of Michigan

Four symposia were devoted to issues in the evaluation of information technologies such as videotex, instructional television, and microcomputers. About half of the sixteen presentations concerned formative evaluation designed to shape the development or implementation of new technologies. The range of applications was quite wide. Don Pelz and Eileen Trzcinski (University of Michigan) looked at the implementation of microcomputers for administrative uses in social service agencies; Rita Bush (AT\&T) examined computerized technologies in the telephone company; Karol Kerns talked about developing electronic "smart cards" to control the distribution of benefits in the Defense Department, and Elise Yoder (Carnegie-Mellon University) described her efforts to refine a computer-aided management system on an aircraft carrier.

Special attention was focused on developing educational technologies for school settings. The newest innovation here is coordinated video images and interactive microcomputer instruction. Saul Rockman (AIT) and Cindy Char (Bank Street College) related the difficulties of doing formative evaluation to develop these multimedia products for schools. Interactive instructional software is difficult enough in a single medium; it is made even more difficult when two media are used. Similar points were made by David Williams and Bill Quinn (Brigham Young University) regarding videodisc/micro instruction at the college level, and by Lisa Ehrlich (Digital Equipment) for developing such instruction for industrial settings.

A number of presentations focused on the impact of the technologies on their users. Shirley Schwartz and her colleagues from Maryland assured us that microcomputer use in preschool does not upset the quality of social play of very young children. Kathline Starr (Bolt, Beranek and Newman) described how microcomputers in elementary classrooms can influence the social life of the classroom in ways that go beyond the direct impact of the educational program students may be 
using. Barbara Davis (University of California, Berkeley) is evaluating videodisc instruction at the college level. She drew some useful lessons from product development-evaluation in the $1960 \mathrm{~s}$. She also noted that the truly cutting-edge technologies are so new that they cannot yet be evaluated; first, evaluators must document how they are being used.

Jim Ettema (University of Minnesota) described a recently completed study of videotex that was experimentally implemented with a group of farmers to test the device's utility as an information tool for agricultural market information. His design used a stakeholder perspective; he showed how a single study could be used to collect information of use to developers, funders, and society at large.

A number of presenters looked broadly at design and measurement issues in the evaluation of the new information technologies. Jim Sanders (Western Michigan University) made the case for studying the context in which the technology is being used, because it can influence so drastically the "meaning" of the hardware and software for the users. $\mathrm{He}$ made a compelling case for lengthy case studies using natural field observation techniques. Henry Ingle (California State University, Chico) showed the merits of video case studies for rapidly evolving phenomena such as microcomputers used in school classrooms. Ron Rice (USC) discussed the newest innovation in data collection: machinegenerated data that come from the very technologies being studied. The data are attractive for their unobtrusive and accurate qualities, but the data are not easily or cheaply managed to provide parsimonious indicators. The biggest challenge they present is their validity. Rice noted that the average correlation between self-report measures of machine use and machine-generated data on the same variable is about 0.40 ! Finally, Jere Johnston (University of Michigan) provided an overview of the characteristics of the new technologies, and then posited some implications these have for research design and analysis. Among other things, he urged that more use be made of case studies and that machine-generated data be used sparingly and only after careful conceptualization of the variables.

Many of the presentations will appear in two future publications. One is an issue of New Directions devoted to general issues in evaluating the new technologies, slated for September 1984. The second is a volume in the Sage Research Progress Series in Evaluation devoted to evaluating technologies designed for educational applications, which also should be available by next fall. 